\section{(2) OPEN ACCESS}

\title{
Fractal analysis of polypoidal choroidal neovascularisation in age-related macular degeneration
}

\author{
Rita Serra (D) , 1,2,3,4 Florence Coscas (D) , ${ }^{3,4}$ Antonio Pinna (D) , ${ }^{5}$ Diogo Cabral, ${ }^{6}$ \\ Gabriel Coscas, ${ }^{3,4}$ Eric H Souied ${ }^{4}$
}

${ }^{1}$ Department of Surgical and Biomedical Sciences, University of Sassari, Sassari, Italy ${ }^{2}$ Istituto di Ricerca Genetica e Biomedica (IRGB), CNR, Cittadella Universitaria di Cagliari, 09042, Monserrato (CA), Cagliari, Italy

${ }^{3}$ Centre Ophtalmologique de I'Odéon, 113 bd Saint Germain, Paris, France

${ }^{4}$ Department of Ophthalmology, Centre Hospitalier Intercommunal de Creteil University Paris Est Créteil, Créteil, France

${ }^{5}$ Department of Medical Surgical, and Experimental Sciences, University of Sassari, Sassari, Italy

${ }^{6}$ Instituto de Oftalmologia Dr. Gama Pinto, Lisboa, Portugal

\section{Correspondence to}

Florence Coscas, Centre Hospitalier Intercommunal de Créteil, Université Paris Est, 40 Avenue Verdun, 94010 Créteil, France; coscas. @gmail.com

Received 27 May 2020 Revised 17 July 2020 Accepted 16 August 2020

\section{Check for updates}

(C) Author(s) (or their employer(s)) 2020. Re-use permitted under CC BY-NC. No commercial re-use. See rights and permissions. Published by BMJ.

To cite: Serra $R$, Coscas $F$ Pinna A, et al. Br J

Ophthalmol Epub ahead of print: [please include Day

Month Year]. doi:10.1136/ bjophthalmol-2020-

317011

\section{ABSTRACT}

Aim To describe optical coherence tomography angiography (OCTA) features of polypoidal choroidal neovascularisation (PCNV) secondary to age-related macular degeneration.

Methods A retrospective consecutive series of 51 patients with a diagnosis of PCNV, based on clinical and multimodal imaging, was analysed. All patients with PCNV underwent a comprehensive ophthalmological examination, including fluorescein and indocyanine green angiography, structural optical coherence tomography (OCT) and OCTA. Two blinded retinal specialists carefully reviewed OCTA slabs in order to assess the morphological patterns of PCNV lesions. Furthermore, fractal analysis of PCNV en face images on OCTA, including vascular perfusion density (VPD), fractal dimension (FD) and lacunarity (LAC), was performed.

Results Fifty-one PCNV eyes were included in the study. In all, the branching vascular network appeared hyperreflective. Polyps showed two different patterns: in 34/51 $(67 \%)$ eyes, they corresponded to hypo-reflective structures, whereas in the remaining 17 (33\%) eyes, they appeared as hyper-reflective lesions. In all PCNV eyes, mean VPD, FD and LAC were $0.76 \pm 0.17 \%, 1.46 \pm 0.12$ and $2.4 \pm 0.87$, respectively. No significant difference was found between PCNVs showing a different OCTA pattern, in terms of quantitative OCTA parameters.

Conclusion Fractal analysis provides quantitative parameters demonstrating that PCNVs with different OCTA patterns share the same neovascular architecture and branching complexity. These new findings improve our ability to interpret OCTA slabs, opening new areas of discussion about this type of neovascular lesion.

\section{INTRODUCTION}

Polypoidal choroidal vasculopathy (PCV), first described by Yannuzzi et al, ${ }^{1}$ is believed to be an acquired abnormal choroidal vasculopathy, characterised by polypoidal dilations (named polyps) arising from terminal ends of a choroidal branching vascular network (BVN) underneath the retinal pigment epithelium (RPE). ${ }^{1} 2$

Recently, two different types of PCVs have been distinguished: type $1 \mathrm{PCV}$, also named 'polypoidal choroidal neovascularisation' (PCNV), located in the sub-RPE space, and type $2 \mathrm{PCV}$, also called 'typical PCV', showing a choroidal localisation. ${ }^{3}$

Even though more commonly detected in pigmented individuals, PCV lesions have been described in all ethnic groups as a recurrent cause of acute vision loss secondary to serous and haemorrhagic pigment epithelium detachment (PED). ${ }^{4}$

Clinically, PCV is characterised by the presence of polypoidal lesions, which usually appear as reddishorange spheroidal subretinal structures arising from choroidal vessels. ${ }^{15}$

On spectral-domain optical coherence tomography (SD-OCT), polypoidal dilatations appear as dome-like RPE elevations ('peaked PED') with moderate internal reflectivity, while the BVN corresponds to the two hyper-reflective lines representing Bruch's membrane and RPE ('double-layer sign'). ${ }^{467}$

Currently, indocyanine green angiography (ICGA) is still recognised as the gold standard for PCV diagnosis, thanks to its ability to detect different structures located underneath the RPE, such as the BVN and polypoidal dilations. ${ }^{8}$ However, ICGA is expensive, time-consuming and associated with the rare, but serious, risk of allergic or anaphylactic reaction. ${ }^{910}$

Recently, OCT angiography (OCTA), a relatively novel non-invasive imaging modality that can generate detailed angiographic images without dye administration in a few seconds, has been introduced in the clinical practice. ${ }^{9} 11$ Thanks to its numerous advantages over ICGA, OCTA is now considered as an important, highly sensitive, non-invasive alternative or adjunct to traditional multimodal imaging in the detection of neovascular lesions secondary to age-related macular degeneration (AMD), PCV included. $^{12-15}$

In particular, in 32 AMD eyes assessed by traditional multimodal imaging and OCTA, the latter technique confirmed the presence of choroidal neovascularisation (CNV) in $81.3 \%$ of cases. ${ }^{12}$ OCTA is also highly sensitive in the detection of inactive $\mathrm{CNV}$, because OCTA visualisation of neovascular lesions is not affected by their activity level and vascular leakage. ${ }^{14} 16 \quad 17$ Similar data have been shown for PCV secondary to AMD. In fact, Huang et $a l^{15}$ reported high OCTA sensitivity rates in the detection of both BVN and polyps (90\% and $86 \%$, respectively), in a cohort of 50 patients.

There is no general agreement on how polypoidal lesions appear on OCTA. Some authors have described them as hypo-reflective aneurysmatic dilations, whereas according to others, they appear as hyper-reflective lesions. ${ }^{13} 1819$ 
Although some theories have been proposed, the reason why PCV may show different features on OCTA scans remains unclear. Recently, fractal analysis of OCTA images has been reported to provide quantitative parameters that are strongly correlated with the OCTA appearance of neovascular lesions in patients with AMD. ${ }^{20} 21$ To the best of our knowledge, we are unaware of any former published study investigating the application of fractal analysis to PCV lesions resistant to intravitreal injections of antivascular endothelial growth factor (VEGF) agents, the current gold standard in the treatment of PCV.

Several reports have demonstrated the ability of anti-VEGF therapy to decrease exudation and improve, or at least stabilise, vision in these patients; however, only minimal changes have been noted in PCV regression, resulting in frequent recurrences of the disease.

Actually, PCV is considered a chronic disorder, needing a continuous, long-term follow-up and treatment. ${ }^{22}$

Therefore, the aim of this study was to report quantitative OCTA parameters of PCNV resistant to anti-VEGF treatment in patients with AMD, in an attempt to better understand the different OCTA presentations of PCNV.

\section{METHODS}

This was a retrospective review of all PCNV cases presented at the Odeon Ophthalmology Center, Paris, France, and the Department of Ophthalmology, University Paris Est, Creteil, France, between 6 January 2015 and 19 April 2017.

This study was conducted in compliance with the tenets of the Declaration of Helsinki for research involving human subjects and was approved by the Paris and Creteil Institutional Review Committees. Included patients signed a written informed consent to participate in this observational study.

For each patient, PCNV diagnosis, based on the presence of the typical BVN and polypoidal dilations on ICGA, ${ }^{1}{ }^{2} 8$ was confirmed by an expert blinded retinal specialist (FC), who meticulously reviewed all clinical and multimodal imaging data, including best corrected visual acuity (BCVA), fluorescein angiography (FA), ICGA (Heidelberg Engineering, Germany), SD-OCT (Heidelberg Engineering) and OCTA.

Exclusion criteria were the presence of any other concomitant ocular disease potentially affecting imaging interpretation (eg, myopia $>6$ D), ocular inflammation, angioid streaks, relevant opacities of the optic media and history of laser photocoagulation and photodynamic therapy.

All patients enrolled in the study underwent a comprehensive ophthalmic examination, including BCVA measured using the ETDRS charts, multimodal imaging evaluation (FA, ICGA and SD-OCT), and OCTA examination using either the swept-source OCTA Triton (Topcon, Tokyo, Japan) or AngioVue XRTVue Avanti (Optovue, Fremont, California, USA).

The $3 \times 3$ angiocube was centred on the PCNV lesions and their appearance was analysed and compared with ICGA. PCNV lesions not entirely included in the OCTA scans were excluded.

Automatic OCTA segmentation was performed by the software embedded in the computer hardware and carefully adjusted in order to better detect blood flow abnormalities suggestive of PCNV and remove immediately segmentation artefacts.

The automatic segmentation provided by the OCTA software was manually adjusted for correct visualisation of the capillary plexus, outer retinal layers and choriocapillaris, in order to better identify the PCNV plane. Furthermore, manual adjustment of the thickness between the two segmentations was necessary to improve PCNV visualisation on the corresponding en face angiogram and immediately remove potential confounders, such as shadowing or projection artefacts.

Then, the location and appearance of each PCNV lesion were analysed by two blinded retinal specialists (RS and FC) and compared with ICGA.

In order to estimate vascular perfusion density (VPD), fractal dimension (FD) and lacunarity (LAC) of the PCNVs, en face OCTA images of the outer retina segmentation were exported into a previously validated custom graphical user interface built in MATLAB (v.r2018a) coding language. Poor quality images with a signal strength index $<60$ and residual artefacts were excluded from the analysis.

Images were binarised using the Otsu method, ${ }^{23}$ while filtering of speckle noise was achieved by using a median filter of radius 2 pixels. Small, non-connected pixels $<10$ were removed. The density map was computed, and the highest density zone was identified. Quantitative OCTA analyses of the blood flow area, VPD, FD and LAC were performed using a graphical interface. The box-counting method at multiple origins was applied to the image of the binary skeleton to estimate the FD and LAC of the vascular network, which are global indices of morphological complexity and structural non-uniformity, respectively. VPD was defined as the total area of perfused vasculature (on the binarised image) per unit area in a region of measurement ${ }^{24}$ (figure 1 ).

The results were then automatically exported into a different file for statistical analyses.

Results of descriptive analysis are expressed as counts and percentages for categorical variables and as mean \pm SD for quantitative variables.

After testing data distribution for normality, paired t-test for continuous variables was performed, as appropriate.

Comparison of mean BCVA between eyes with hypo-reflective and hyper-reflective polyps was performed using the Student's t-test. A p value $<0.05$ was considered to be statistically significant.

The study data were analysed using the Statistical Package for Social Sciences version 20.0 for Mac (IBM, Chicago, IL).

\section{RESULTS}

A total of 51 patients (31 males, 20 females; mean age 71.73 \pm 12.31 years) was included in the study. Eleven patients were enrolled at the Odeon Ophthalmology Center in Paris, France, whereas the remaining 40 were recruited at the Department of Ophthalmology of the University of Paris Est, Creteil, France.

In $33 / 51(64.7 \%)$ eyes, PCNVs were located in the macular region, while only $18 / 51$ (35.3\%) eyes showed PCNVs close to the optic disc.

SD-OCT revealed the presence of subretinal fluid in $40 / 51$ (78.4\%) eyes, of whom seven had an associated haemorrhagic component. Intraretinal fluid was observed in 2/51 (4\%) eyes; the remaining 9/51 (17.6\%) showed both subretinal and intraretinal fluid.

In all cases, early ICGA phase revealed a hyperfluorescent neovascular network corresponding to the BVN with peripheral aneurysmal dilations.

Comparison of mid/late ICGA and OCTA revealed a perfect correspondence in shape and location between the hyperfluorescent BVN on ICGA and the hyper-reflective network on OCTA, appearing as a flat, hyper-reflective PED on simultaneous structural OCT.

Additionally, polypoidal lesions appearing as aneurysmatic hyperfluorescent dilations on ICGA perfectly corresponded to roundish hypo-reflective structures in 34/51 (67\%) eyes and 


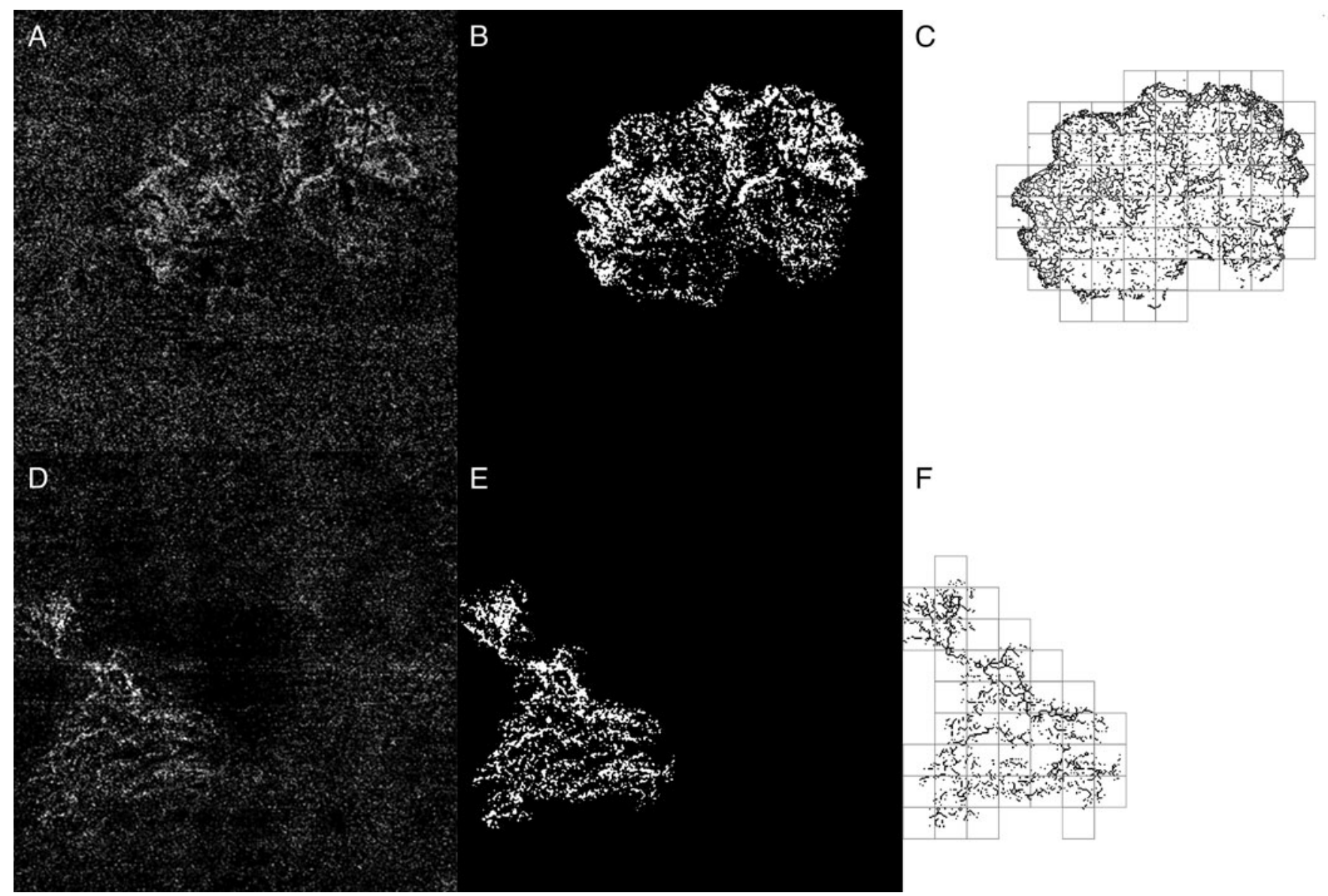

Figure 1 Representation of the algorithm used to measure quantitative polypoidal choroidal neovascularisation parameters, on optical coherence tomography angiography images. (A, D) En face outer retina images were exported and then imported into a custom graphical user interface built in MATLAB (v.r2018a) coding language. (B, E) Then, images were binarised using the Otsu method. The speckle noise was filtered to identify the vascular network and thus estimate the vascular perfusion density. (C, F) Finally, images were skeletonised to estimate fractal dimension and lacunarity by boxcounting method, using the graphical interface.

hyper-reflective lesions in 17 (33\%) eyes, on OCTA slabs of the outer retina.

Representative images of different OCTA patterns of PCNV are shown in figures 2 and 3 .

All OCTA findings were matched with the results of multimodal imaging, so as to be sure of the correct location of the PCNV during segmentation analysis.

All eyes were previously treated with intravitreal anti-VEGF agents (mean 5.04 \pm 1.56 intravitreal injections).

Mean BCVA was 83.22 \pm 14.91 ETDRS letters, and no difference was found in terms of mean BCVA between eyes showing polyps with a different OCTA appearance (hypo-reflective vs hyper-reflective polyps).

In PCNV eyes, mean VPD was $0.76 \pm 0.17 \%$, mean FD 1.46 \pm 0.12 and mean LAC $2.4 \pm 0.87$. No statistical difference was found between the two PCNV patterns for all fractal parameters analysed. All demographic characteristics and quantitative OCTA findings are summarised in tables 1 and 2, respectively.

\section{DISCUSSION}

In the last few years, the spread of OCTA in the clinical practice has improved our ability to study PCNV, increasing the understanding of this neovascular disorder. 111315181925

Indeed, some investigations have assessed the threedimensional structure of PCNV, in an attempt to establish the exact retinal location of the different PCNV components. ${ }^{25} 26$
These OCTA studies have suggested that polyps and BVN are situated in the compartment space between the RPE and Bruch membrane, rather than in the inner choroid, as previously hypothesised on the basis of traditional clinical findings and angiographic interpretations. ${ }^{26} 27$ Other reports have detailed the morphological appearance of PCNV secondary to AMD but showed conflicting results. Actually, although en face angiograms clearly show BVN as a hyper-reflective lesion corresponding to a flat PED in structural OCT, the OCTA appearance of polyps remains still debatable. ${ }^{13} 1819$

Kim et $a l^{18}$ evaluated a cohort of 24 eyes with PCNV and rated them as hypo-reflective aneurysmatic dilatations in 50\% of cases. Similarly, in a study on $12 \mathrm{PCNV}$ eyes, Srour et al ${ }^{13}$ described the polyps as roundish hypo-reflective structures at the edge of the BVN in nine eyes and as hyper-reflective lesions in the remaining three. Conversely, in a cohort of 20 Asiatic patients assessed with swept-source OCTA, Bo et al ${ }^{19}$ observed that all polyps appeared as densely or loosely tangled vessels before and after treatment, respectively, rather than aneurysmatic structures.

Some disagreement on how the polypoidal lesions appear on OCTA was also found in our survey. Although the BVN appeared as a hyper-reflective network on the outer retina OCTA segmentation in all the affected eyes, polyps appeared as hypo-reflective aneurysmatic structures in 34/51 (67\%) eyes and as hyperreflective lesions in the remaining 17 (33\%). 


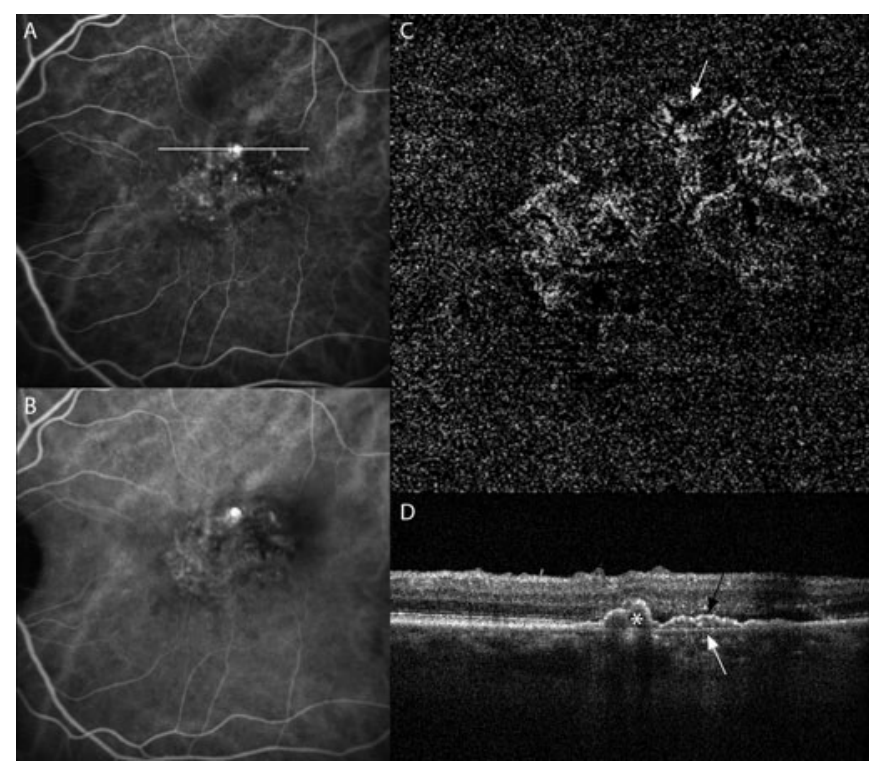

Figure 2 Left eye: multimodal imaging and optical coherence tomography angiography of a polypoidal choroidal neovascularisation, secondary to age-related macular degeneration. (A, B) Early- and mid-frame of indocyanine green angiography showing the presence of a hyperfluorescent vascular network suggestive of branching vascular network (BVN) with a roundish hyperfluorescent lesion suggestive of a polyp, arising at the terminal ends. (C) En face angiogram of the outer retina segmentation shows a hyper-reflectiveneovascular network suggestive of the BVN with a hypo-reflective roundish region at theterminal ends corresponding to a hypo-reflective polyp (white arrow). (D) Spectral-domain optical coherence tomography reveals the presence of double-layersign, represented by two hyper-reflective lines, inner signifying shallow retinal pigmentepithelium detachment (PED) (black arrow), and outer signifying Bruch's membrane (whitearrow) indicative of BVN and a peaked PED corresponding to the polyp (white asterisk). Note the hypo-reflective area suggesting a subretinal fluid accumulation (see white line inA).

The reason why polyps show different features on OCTA is still a matter of debate. It is important to note that the ability of OCTA to visualise the blood flow within retinal vessels is limited to a certain range of flow velocities (minimum: $0.5-2 \mathrm{~mm} / \mathrm{s}$, saturation: $9 \mathrm{~mm} / \mathrm{s}$ estimated for current devices). Theoretically, some neovascular networks or parts of them may have a flow speed below the OCTA detection limit. ${ }^{28}$ In such a condition, polyps may appear as hypo-reflective lesions.

Rebhun et al, ${ }^{29}$ using a novel OCTA algorithm termed Variable Interscan Time Analysis, have recently demonstrated the presence of variable flow speeds in the different PCNV components and also within the single polyp. In particular, a range of fast/moderate blood flow speeds was found in the BVN, whereas polyps were characterised by slow flow speeds. Interestingly, some polyps showed two different flow speeds within the same lesion, one with a faster and another with a slower flow, thus suggesting a turbulent flow within the polyp, which may account for the absence of OCTA signal in most of them. ${ }^{12}$ This theory has also been used to explain the OCTA appearance of diabetic retinopathy microaneurysms, which shares several features with PCNV, including the turbulent flow within the lesion and the hyporeflectivity on OCTA. ${ }^{30}$

Traditionally, the belief that polyps have a turbulent blood flow comes from the phenomenon of dye washout and pulsatile blood flow observed on ICGA. ${ }^{31} 32$ However, this ICGA feature is not

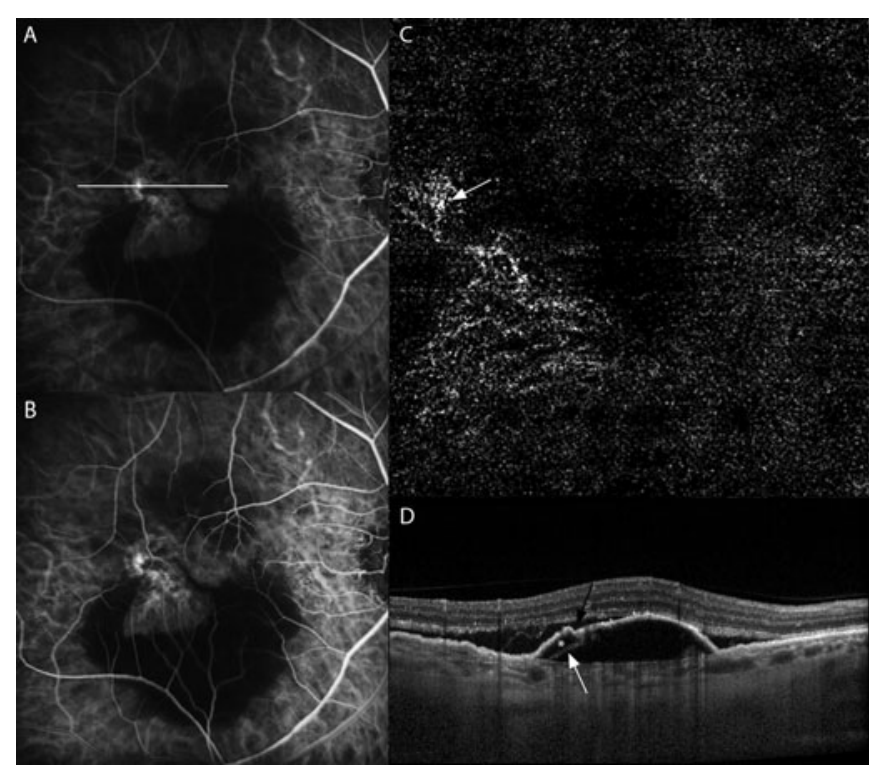

Figure 3 Right eye: multimodal imaging and optical coherence tomography angiography of a polypoidal choroidal neovascularisation, secondary to age-related macular degeneration. (A, B) Early- and mid-frame of indocyanine green angiography showing the presence of a hyperfluorescent vascular network suggestive of branching vascular network with a hyperfluorescent lesion suggestive of a polyp. (C) En face angiogram of the outer retina segmentation shows a hyper-reflective neovascular network suggestive of the BVN with a hyper-reflective region corresponding to a hyper-reflective polyp (white arrow). (D) Spectraldomain optical coherence tomography reveals the presence of a notched retinal pigment epithelium detachment (PED). Note the notch of the PED (black arrow), along with hypo-reflective lumen (white asterisk) surrounded by hype-reflective ring (white arrow) attached to undersurface of retinal pigment epithelium (see white line in A).

detectable in all PCNV eyes. On the basis of OCTA analysis, it has been hypothesised that the turbulent blood flow observed in polyps may be caused by the presence of vascular dilations and changes in vascular calibre and direction within the lesion. ${ }^{19}$

Recently, some authors have postulated that quantitative OCTA parameters obtained by fractal analysis of en face angiograms, such as VPD, FD and LAC, could be reliable biomarkers for an objective assessment of the clinical activity, natural history and prognosis of neovascular lesions secondary to AMD. ${ }^{20} 21$ Serra et $a l^{20}$ have demonstrated that FD and LAC are useful biomarkers to distinguish type $1 \mathrm{CNV}$ s with different natural history and prognosis. Similarly, Al-Sheik et $a l^{21}$ claimed that FD may be a potential biomarker to assess type $1 \mathrm{CNV}$ clinical activity. However, in both studies, ${ }^{20} 21$ there was no evidence of fractal analysis of OCTA slabs performed in eyes showing PCNV secondary to AMD.

In our investigation, VPD, FD and LAC were $0.76 \pm 0.17 \%$, $1.46 \pm 0.12$ and $2.4 \pm 0.87$, respectively. Comparing our results with previously published data, ${ }^{21}$ active PCNVs seem to show higher VPD and lower FD values than those found in active type 1 $\mathrm{CNV}$, a finding suggesting that the neovascular architecture in PCNVs is different from that in type $1 \mathrm{CNV}$.

PCNV and type $1 \mathrm{CNV}$ share many similarities in terms of therapy and prognosis; therefore, according to the latest research and classification, PCNV has been included in the type I CNV group. ${ }^{33}$ Conversely, our fractal analysis data seem to support the idea that PCNVs are separate entities, rather than variants of occult CNVs. ${ }^{33}$ 
Table 1 Demographic features of polypoidal choroidal neovascularization patients showing polyps with a different appearance on optical coherence tomography angiography $(n=51)$

\begin{tabular}{lll}
\hline & $\begin{array}{l}\text { PCNV with } \\
\text { hypo-reflective polyps }\end{array}$ & $\begin{array}{l}\text { PCNV with } \\
\text { hyper-reflective polyps }\end{array}$ \\
\hline $\begin{array}{l}\text { Total eyes, } \mathrm{n}(\%) \\
\text { Sex }\end{array}$ & $34(67)$ & $17(33)$ \\
Male, $\mathrm{n}(\%)$ & $19(55.8)$ & $12(70.5)$ \\
- Female, $\mathrm{n}(\%)$ & $15(44.2)$ & $5(29.5)$ \\
$\begin{array}{l}\text { Age, mean } \pm \text { SD (years) } \\
\begin{array}{l}\text { BCVA, mean } \pm \text { SD (ETDRS } \\
\text { letters) }\end{array}\end{array}$ & $74.45 \pm 12.56$ & $68 \pm 11.29$ \\
\hline
\end{tabular}

Categorical variables are presented as $\mathrm{n}(\%)$.

Continuous variables are presented as mean \pm SD.

BCVA, best corrected visual acuity; PCNV, polypoidal choroidal neovascularisation.

Table 2 Quantitative optical coherence tomography angiography (OCTA) parameters of polypoidal choroidal neovasculariszation eyes showing polyps with a different OCTA appearance

\begin{tabular}{llll}
\hline & $\begin{array}{l}\text { PCNV with } \\
\text { hypo-reflective } \\
\text { polyps }\end{array}$ & $\begin{array}{l}\text { PCNV with } \\
\text { hyper-reflective } \\
\text { polyps }\end{array}$ & P value \\
\hline VPD, mean \pm SD $(\%)$ & $0.73 \pm 0.17$ & $0.80 \pm 0.15$ & $>0.05$ \\
FD, mean \pm SD & $1.47 \pm 0.12$ & $1.46 \pm 0.12$ & $>0.05$ \\
LAC, mean \pm SD & $2.81 \pm 0.98$ & $2.30 \pm 0.76$ & $>0.05$ \\
\hline
\end{tabular}

Continuous variables are presented as mean \pm SD.

FD, fractal dimension; LAC, lacunarity; PCNV, polypoidal choroidal neovascularisation; VPD, vascular perfusion density.

Although the first description of PCNV dates back to more than 30 years ago, ${ }^{1}$ whether $\mathrm{PCNV}$ is a variant of $\mathrm{CNV}$ secondary to AMD, or a distinct entity, is still a matter of debate. ${ }^{34}$ The aim of our study was simply to report quantitative OCTA parameters in PCNV and not to compare PCNV with type $1 \mathrm{CNV}$. Therefore, future studies are necessary to resolve this actual controversy in clinical retinal research.

After comparing BCVA and quantitative parameters of eyes with different OCTA patterns of PCNV, we failed to find any statistical difference. This result suggests that PCNVs with different OCTA patterns are likely to share the same neovascular organisation and branching complexity. However, we cannot rule out that the different OCTA appearance of polyps might be somehow related to shadowing artefacts. Noteworthy, the more superficial retinal layers and vessels may be falsely detected in the deeper layers because of OCTA projection artefacts. Indeed, since transmitted light diffuses through flowing blood, the deeper layers are reached by fluctuating light, which can create a decorrelation between two successive B-scans at the same location. ${ }^{35}$ Furthermore, RPE and Bruch membrane alterations, relatively common in patients treated with anti-VEGF agents, may contribute to the hyper-reflective polyp appearance on OCTA scans, due to lower blockade of the light source by RPE. Spaide $e t a l^{36}$ have clarified that the signal from the choroid is low, in comparison to the retina, as light is lost because of scattering and attenuation by RPE and the dense microvasculature in the choriocapillaris. In fact, it is well known that choroidal vessels appear white in areas of RPE atrophy, but they are black in areas with normal RPE. ${ }^{37}$

Therefore, this raises the interesting questions as to whether treatment-naïve PCNVs show similar features on OCTA.

Our study has several limitations, mainly due to its retrospective nature and the relatively small number of eyes analysed.
Furthermore, it is important to acknowledge that the presence of a large serosanguinous PED, haemorrhage or extensive exudation, typically seen in eyes with active $\mathrm{PCNV}^{1}$ might have somehow influenced fractal analysis results. Last, but not least, we used two different OCTA devices to evaluate PCNV eyes.

As far as we know, this is the first investigation performing fractal analysis on en face angiograms of PCNV secondary to AMD and reporting quantitative OCTA features of PCNV eyes. Furthermore, on the basis of OCTA fractal analysis results, we have demonstrated that PCNVs showing a different appearance on OCTA share the same neovascular organisation and branching complexity and cause equal visual loss. These new findings improve our understanding of PCNV appearance on OCTA, potentially increasing our ability to interpret OCTA slabs and opening new areas of discussion about this type of neovascular lesion.

Correction notice This paper has been amended since it was published online. The first author's name was incorrectly transposed.

Acknowledgements The authors are sincerely grateful to Dr Sergio Pilia for his assistance in images processing.

Contributors RS: research design, data acquisition, data analysis, manuscript preparation. FC: data acquisition, data analysis, research design, manuscript preparation. AP: data analysis, manuscript preparation. DC: data acquisition, data analysis. GC and EHS: research design, manuscript preparation.

Funding The authors have not declared a specific grant for this research from any funding agency in the public, commercial or not-for-profit sectors.

\section{Competing interests None declared.}

Ethics approval This study was conducted in compliance with the tenets of the Declaration of Helsinki for research involving human subjects and was approved by the Paris and Creteil Institutional Review Committees (IRCS). No number/ID of the approval is provided because this was a retrospective study conducted on already available data. Included patients signed a written informed consent to participate in this observational study.

Provenance and peer review Not commissioned; externally peer reviewed.

Data availability statement All data relevant to the study are included in the article or uploaded as supplemental information.

Open access This is an open access article distributed in accordance with the Creative Commons Attribution Non Commercial (CC BY-NC 4.0) license, which permits others to distribute, remix, adapt, build upon this work non-commercially, and license their derivative works on different terms, provided the original work is properly cited, appropriate credit is given, any changes made indicated, and the use is noncommercial. See: http://creativecommons.org/licenses/by-nc/4.0/.

\section{ORCID iDs}

Rita Serra http://orcid.org/0000-0002-6341-1435

Florence Coscas http://orcid.org/0000-0002-9888-5040

Antonio Pinna http://orcid.org/0000-0003-3052-2662

\section{REFERENCES}

Yannuzzi LA, Sorenson J, Spaide RF, et al. Idiopathic polypoidal choroidal vasculopathy (IPCV). Retina 1990;10:1-8

2 Ciardella AP, Donsoff IM, Huang SJ, et al. Polypoidal choroidal vasculopathy. Surv Ophthalmol 2004;49:25-37

3 Tanaka K, Mori R, Kawamura A, et al. Comparison of OCT angiography and indocyanine green angiographic findings with subtypes of polypoidal choroidal vasculopathy Br J Ophthalmol 2017:101:51-5.

4 Anantharaman G, Sheth J, Bhende M, et al. Polypoidal choroidal vasculopathy: pearls in diagnosis and management. Indian J Ophthalmol 2018;66:896-908.

5 Ampornpruet A, Ruamviboonsuk P, Kokekhuntod S. The fundoscopic features for differentiation between polypoidal choroidal vasculopathy and choroidal neovascularization from age-related macular degeneration. J Med Assoc Thai 2005;88:S51-S56

6 Otsuji T, Takahashi K, Fukushima I, et al. Optical coherence tomographic findings of idiopathic polypoidal choroidal vasculopathy. Ophthalmic Surg Lasers 2000:31:210-4.

7 Sato T, Kishi S, Watanabe G, et al. Tomographic features of branching vascular networks in polypoidal choroidal vasculopathy. Retina 2007;27:589-94.

8 Spaide RF, Yannuzzi LA, Slakter JS, et al. Indocyanine green videoangiography of idiopathic polypoidal choroidal vasculopathy. Retina 1995;15:100-10. 
9 Coscas F, Lupidi M, Boulet JF, et al. Optical coherence tomography angiography in exudative age related macular degeneration: a predictive model for treatment decisions. Br J Ophthalmol 2019;103:1342-6.

10 Hope-Ross M, Yannuzzi LA, Gragoudas ES, et al. Adverse reactions due to indocyanine green. Ophthalmology 1994;101:529-33.

11 Nagiel A, Sadda SR, Sarraf D. A promising future for optical coherence tomography angiography. JAMA Ophthalmol 2015;133:629-30.

12 Faridi A, Jia Y, Gao S, et al. Sensitivity and specificity of OCT angiography to detect choroidal neovascularization. Retina 2017;1:294-303.

13 Srour M, Querques G, Semoun 0, et al. Optical coherence tomography angiography characteristics of polypoidal choroidal vasculopathy. $\mathrm{Br} J$ Ophthalmol 2016;100:1489-93.

14 Ichiyama T, Sawada T, Ito Y, et al. Optical coherence tomography angiography reveals blood flow in choroidal neovascular membrane in remission phase of neovascular age-related macular degeneration. Retina 2017;37:724-30.

15 Huang CH, Yeh PT, Hsieh YT, et al. Characterizing branching vascular network morphology in polypoidal choroidal vasculopathy by optical coherence tomography angiography. Sci Rep 2019;9:595.

16 Serra R, Coscas F, Boulet JF, et al. Predictive activation biomarkers of treatment-naïve asymptomatic choroidal neovascularization in age-related macular degeneration. Retina 2019.

17 Forte R, Coscas F, Serra R, et al. Long-term follow-up of quiescent choroidal neovascularization associated with age-related macular degeneration or pachychoroid disease. Br J Ophthalmol 2019.

$18 \mathrm{Kim}$ JY, Kwon OW, Oh HS, et al. Optical coherence tomography angiography in patients with polypoidal choroidal vasculopathy. Graefes Arch Clin Exp Ophthalmol 2016;254:1505-10

19 Bo Q, Yan Q, Shen M, et al. Appearance of polypoidal lesions in patients with polypoidal choroidal vasculopathy using swept source optical coherence tomography angiography. JAMA Ophthalmol 2019;137:642-50.

20 Serra R, Coscas F, Pinna A, et al. Quantitative optical coherence tomography angiography features of inactive macular neovascularization in age-related macular degeneration. Retina 2020; Publish Ahead of Print.

21 Al-Sheikh M, lafe NA, Phasukkjiwatana N, et al. Biomarkers of neovascular activity in age-related macular degeneration using optical coherence tomography angiography. Retina 2018;38:220-30.

22 Palkar AH, Khetan V. Polypoidal choroidal vasculopathy: an update on current management and review of literature. Taiwan J Ophthalmol 2019;9:72-92.

23 Otsu N. A threshold selection method from gray-level histograms. IEEE Trans Syst Man Cybern 1979;9:62-6.
24 Durbin MK, An L, Shemonski ND, et al. Quantification of retinal microvascular density in optical coherence tomography angiography images in diabetic retinopathy. JAMA Ophthalmol 2017;135:370-6.

25 Tomiyasu T, Nozaki M, Yoshida M, et al. Characteristics of polypoidal choroidal vasculopathy evaluated by optical coherence tomography angiography. Invest Ophthalmol Vis Sci 2016;57:0CT324-0CT330.

26 Chi YT, Yang CH, Cheng CK. Optical coherence tomography angiography for assessment of the 3-dimensional structures of polypoidal choroidal vasculopathy. JAMA Ophthalmol 2017;135:1310-16.

27 Wang M, Zhou Y, Gao SS, et al. Evaluating polypoidal choroidal vasculopathy with optical coherence tomography angiography. Invest Ophthalmol Vis Sci 2016;57: OCT526-OCT532.

28 Lindner M, Fang PP, Steinberg JS, et al. OCT angiography-based detection and quantification of the neovascular network in exudative AMD. Invest Ophtha/mo/ Vis Sci 2016:57:6342-8.

29 Rebhun CB, Moult EM, Novais EA, et al. Polypoidal choroidal vasculopathy on swept-source optical coherence tomography angiography with variable interscan time analysis. Trans/ Vis Sci Technol 2017;6:4.

30 Matsunaga DR, Yi JJ, De Koo LO, et al. Optical coherence tomography angiography of diabetic retinopathy in human subjects. Ophthalmic Surg Lasers Imaging Retina 2015;46:796-805

31 Akaza E, Matsumoto Y, Yuzawa M. Pulsation in polypoidal choroidal vasculopathy. Nippon Ganka Gakkai Zasshi 2006;110:288-92.

32 Chen Y, Yang Z, Xia F, et al. The blood flow characteristics of polypoidal choroida vasculopathy and the choroidal remodelling process after photodynamic therapy. Lasers Surg Med 2018:50:427-32.

33 Spaide RF, Jaffe GJ, Sarraf D, et al. Consensus nomenclature for reporting neovascular age-related macular degeneration data: consensus on neovascular age related macular degeneration nomenclature study group. Ophthalmology 2020;127:616-36

34 Laude A, Cackett PD, Vithana EN, et al. Polypoidal choroidal vasculopathy and neovascular age-related macular degeneration: same or different disease? Prog Retin Eye Res 2010:29:19-29.

35 Spaide RF, Fujimoto JG, Waheed NK, et al. Optical coherence tomography angiography. Prog Retin Eye Res 2018;64:1-55.

36 Spaide RF, Fujimoto JG, Waheed NK. Image artifacts in optical coherence tomography angiography. Retina 2015;35:2163-80.

37 Maruko I, Koizumi H, Sawaguchi S, et al. Choroidal blood vessels in retinal pigment epithelial atrophy using optical coherence tomography angiography. Retin Cases Brief Rep 2019;13:88-93. 